\begin{tabular}{|c|c|}
\hline Title & Detection of Bibliographic Coupling Communities U sing Research Output (2004 2013) from Nepal \\
\hline Author(s) & Gautam, Pitambar \\
\hline Citation & $\begin{array}{l}2017 \text { 6th IIA I International Congress on A dvanced A pplied Informatics (IIA I-A A I), 202-207 } \\
\text { https:/doi.org/10.1109/IA I-A A I.2017.72 }\end{array}$ \\
\hline Issue Date & $2017-07$ \\
\hline Doc URL & http:/hdl.handle.net/2115/67641 \\
\hline Rights & $\begin{array}{l}\text { () } 2017 \text { IEEE. Personal use of this material is permitted. Permission from IEEE must be obtained for all other uses, in } \\
\text { any current or future media, including reprinting/republishing this material for advertising or promotional purposes, } \\
\text { creating new collective works, for resale or redistribution to servers or lists, or reuse of any copyrighted component of } \\
\text { this work in other works. }\end{array}$ \\
\hline Type & proceedings (author version) \\
\hline Note & $\begin{array}{l}\text { IIA I International Congress on A dvanced A pplied Informatics 2017 II A I A A I 2017. 9-13 July 2017. Hamamatsu, } \\
\text { Shizuoka, Japan. }\end{array}$ \\
\hline File Information & gautam-dsir2017final.pdf \\
\hline
\end{tabular}

Instructions for use 


\title{
Detection of Bibliographic Coupling Communities Using Research Output (2004-2013) From Nepal
}

\author{
Pitambar Gautam \\ Creative Research Institution (CRIS) \\ Hokkaido University \\ N21 W10, Kita Ku, Sapporo, Hokkaido 001-0021, Japan \\ e-mail: pgautam@cris.hokudai.ac.jp
}

\begin{abstract}
This study explores a country-level bibliometric analysis to extract bibliographic coupling (BC) communities as clusters of documents coupled through publications in their reference lists using the "BiblioTool" software. The 2004-2013 research output from Nepal represented by relatively small dataset of 3,011 documents (peer reviewed articles and reviews) indexed as core collections in the Web of Science (WoS) database was used. Setting a threshold of 10 documents, twenty-five BC communities, each with 12-443 documents, which provide a comprehensive picture on the research themes characterized by diverse items (keywords, subjects, journals, institutions, countries, authors, references, and title words) were discriminated. Twelve communities (i.e., $48 \%$ ) deal with medical \& health sciences (maternal \& child health; tropical infectious diseases; cancer \& cardiovascular diseases; mountain sickness; blindness) closely linked also with social aspects; 4 communities with earth, environment and biodiversity (tectonics and natural hazards; environmental pollution, remediation and conservation; wild-life preservation); 3 communities with agriculture and veterinary sciences (pathogens of major crops (maize, wheat, rice) \& crop yields, plant genes, and dairy farming); 3 communities with nanomaterials and metal-alloys; 2 communities with pharmacology including ethnomedicine, and one community of 12 documents is related to galactic observations. These new results provide wider insights on the research volume \& diversity, international collaboration and the contributors (academic, national \& international, governmental \& nonprofit agencies, etc.) engaged in research in Nepal.
\end{abstract}

Keywords- bibliometrics; scientometry; research community; Web of Science; bibliographic coupling; Nepal; higher education; international co-authorship, developing country

\section{BACKGROUND AND RESEARCH PROCEDURE}

A bibliometric analysis on Nepal's research output recorded in the Web of Science (WoS) core collections [1] in SCI-E, SSCI and A\&HCI databases since 1955 suggests (i) a very low volume, negligible growth and lack of distinct trends until 1989, ii) a marked growth followed by stagnation linked to political instability during the next 15 years, and (iii) recovery and accelerated growth thereafter [2]. It compares two subsequent 10-yrs periods (1994-2003 and 2004-2013), coinciding roughly with political insurgency and followed stability and marked by a 3-fold increase in scientific output during the latter [2]. Disciplinary profiles using the Essential Science Indicators (ESI) 22 subject areas, semantic map of nouns and noun phrases in the text, and citation-based impact indicators (category normalized citation impact (CNCI) and data on the percentage of top $10 \%$ papers (PP Top10)) as well as institutional/international co-authorship relationships for 2004-2013 publications (3,011 articles and reviews) obtained are summarized in Table I. Figure 1 reproduces partly those data for peerreviewed articles \& reviews with at least one author affiliation in Nepal and extends to 2016.

Comparison of the above-mentioned findings with Scopus-based interpretation [3] for 2006-2010, a period common to both studies, reveals that the latter has captured roughly 2.5 times more documents dominated by 'medicine', 'earth and environmental sciences' and 'agro-biological sciences' as found in the former, along with 'life sciences' subject areas (biochemistry, genetics \& molecular biology; immunology \& microbiology) to some extent. One major difference between the two datasets was the journal coverage: while the Journal of the Nepal Medical Association seemed to be the only journal from Nepal indexed in WoS, the Scopus's list was more extensive with additional titles such as Kathmandu University Medical Journal, Nepal Medical College Journal, Journal of the Nepal Paediatric Society, Nepalese Journal of Ophthalmology.

The disciplinary analysis mentioned was limited to coarse disciplinary classifications (ESI 22 and Scopus ASJC 27). Because of the differing journal to discipline assignment practices (single ESI field based on journal carrying the document, but often multiple ASJC subject areas for single document based also on journal) and lack of any established relationships among the disciplines, the results derived can be compared only in broad qualitative terms [4]. The inclusion of citation indicators in the WoS-based analysis helped to identify the broad disciplines with high citation impact and the status in each field relative to the world average, while the semantic map effectively revealed the most prolific and broad cross-disciplinary research areas in which Nepali and foreign researchers are involved actively. However, the methodology used so far is thought to be inadequate for identification of the narrow but niche research areas that reflect the diversity of research down to intermediate scale. 
In order to get further insights at finer disciplinary levels, and a comprehensive picture on the research communities in terms of diverse items other than the disciplines and citations, this study attempts to explore the bibliographic coupling (BC) method [5] to extract the BC communities. There are 3 reasons to use this method: (i) Possibility to use all documents irrespective of whether they were cited or not after publication; (ii) Incorporation of a finer-scale disciplinary classification (i.e., WoS 251 subject category (SC) scheme), (iii) Possibility to extract the results as a set of community cards characterized by diverse items (author and publisher keywords, subject categories (WoS SCs), publication sources, institutions of affiliation of authors, publication sources in references, and countries of locations of institutions) characterizing a large number of research themes/topics, and (iii) freely available BiblioTool software [6] for rapid analysis. As the aim of this study is to explore the potential of bibliometric data in understanding additional aspects of the past research landscape, this renewed study uses the same 2004-2013 dataset described above.

TABLE I. SUMMARY OF THE FIRST STAGE BIBLIOMETRIC ANALYSIS OF THE 2004-2013 PUBLICATIONS FROM NEPAL [2]

\begin{tabular}{|c|c|}
\hline $\begin{array}{l}\text { Type of } \\
\text { analysis }\end{array}$ & Results obtained with plausible interpretation \\
\hline $\begin{array}{l}\text { Disciplinary } \\
\text { coverage }\end{array}$ & $\begin{array}{l}\text { Seven prolific fields with >80\% share of total publications: Clinical Medicine (40\%), Plant \& Animal Science and } \\
\text { Environment/Ecology (10\% each), Social Sciences (8\%), Geosciences and Agricultural sciences (5\% each) and } \\
\text { Chemistry (slightly less than 4\%) }\end{array}$ \\
\hline $\begin{array}{l}\text { Citation impact } \\
\text { (CI: CNCI or } \\
\text { PPTop10) }\end{array}$ & $\begin{array}{l}\text { Among the prolific fields, CI exceeds the world average for Geosciences, Clinical Medicine and Agricultural } \\
\text { Sciences, but it is well below the world average for Social Sciences, Chemistry, Environment/Ecology and Plant } \\
\& \text { Animal Science }\end{array}$ \\
\hline & $\begin{array}{l}\text { Among the less prolific }(0.7-2.2 \% \text { share) fields, CI is distinctly above the world average for Molecular Biology \& } \\
\text { Genetics, Economics \& Business, Psychiatry \& Psychology, Materials Science and Biology \& Biochemistry, but } \\
\text { it is close to the world average for Pharmacology \& Toxicology, Microbiology, and Immunology }\end{array}$ \\
\hline \multirow{7}{*}{$\begin{array}{l}\text { International co- } \\
\text { authorship (IC) }\end{array}$} & A high overall average $(76.4 \%)$ \\
\hline & $\begin{array}{l}\text { Top ten contributing countries besides Nepal: USA (19.6\%); India (15.3\%); UK (11.4\%); Japan (10.8\%); South } \\
\text { Korea (5.8\%); Germany (4.7\%); Peoples R China (4.4\%); Australia (4.1\%); Switzerland (3.8\%); Thailand (3.8\%) }\end{array}$ \\
\hline & $\begin{array}{l}\text { Top } 10 \text { contributing domestic or foreign institutions: Tribhuvan Univ (29.1\%); BP Koirala Inst Hlth Sci (11.1\%); } \\
\text { Kathmandu Univ (5.1\%); Int Ctr Integrated Mt Dev (3.9\%); Johns Hopkins Univ (3.4\%); Manipal Coll Med Sci } \\
\text { Nepal (3.3\%); Banaras Hindu Univ (3\%); Ctr Int Mejoramiento Maiz \& Trigo (2.6\%); Inst Trop Med Antwerp } \\
\text { (2.4\%); Res Lab Biotechnol \& Biochem Nepal (2.4\%) }\end{array}$ \\
\hline & $\begin{array}{l}\text { Lowest for Clinical Medicine }(56.5 \%) \text { and Neuroscience \& Behavior }(61.1 \%) \text {, indicating that domestic institutions } \\
\text { are in relatively better position to carry independent research in these fields }\end{array}$ \\
\hline & $\begin{array}{l}\text { Highest in Molecular Biology \& Genetics and Space Science (100\%) indicating that Nepal is least equipped to } \\
\text { conduct independent research }\end{array}$ \\
\hline & Very high (91-96\%) for Geosciences, Agricultural Sciences, and Chemistry and high (78-83\%) for \\
\hline & $\begin{array}{l}\text { Environment/Ecology, Social Sciences (general), Plant \& Animal science, implying that a lot still required to } \\
\text { develop research capacity of Nepal-based researchers }\end{array}$ \\
\hline $\begin{array}{l}\text { Research topics } \\
\text { from semantic } \\
\text { maps }\end{array}$ & $\begin{array}{l}\text { Six research clusters (only major, because of analysis targeted at visualization), mostly integrative/cross- } \\
\text { disciplinary: (i) Child \& maternal health, family planning; (ii) Clinical medicine, tumour, mountain sickness; } \\
\text { (iii) Infectious diseases; (iv) Agroforestry; (v) Wheat research, and (vi) Protein structure analysis \& synthesis }\end{array}$ \\
\hline
\end{tabular}

Figure 1. Annual trend of publications with at least one author affiliation in Nepal (solid circles) showing an exponential increase with time (fitted solid curve) along with the annual publications increment (vertical bars). Data retrieved from the Web of Science database [1]

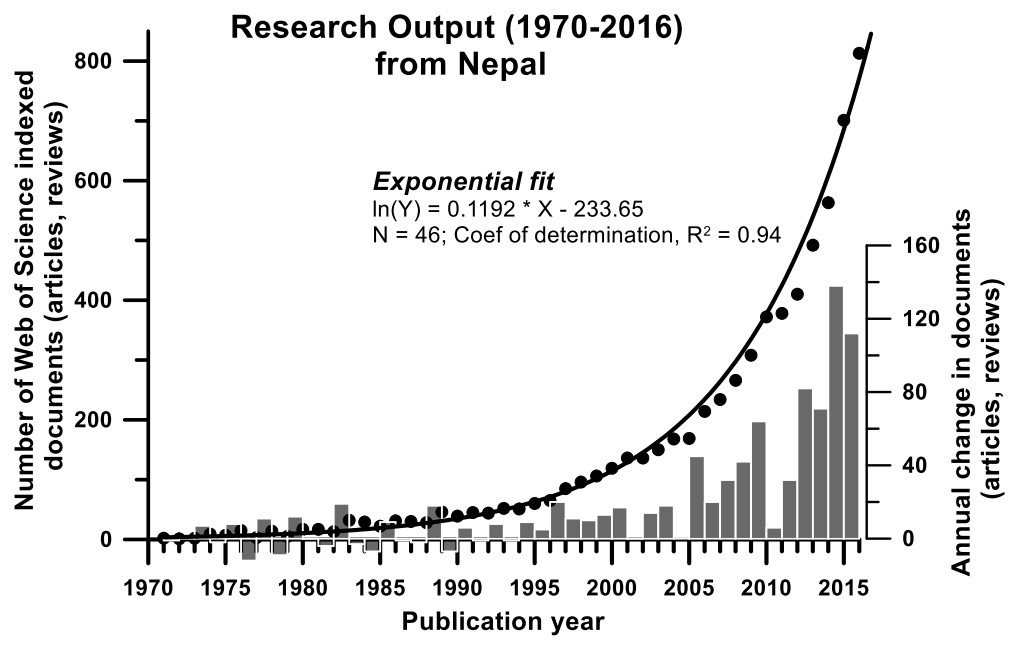


The bibliographic data for 3,011 documents extracted from WoS as windows tab-delimited files were analyzed in 2 steps. First, these files were parsed into separate subsets of items (articles, authors, countries, institutions, keywords, references, subject categories and title-words) using the BiblioTool 2.3 python routines [6]. The dataset for institutions was subjected to rigorous "cleaning" with emphasis on unification of all possible variants of the affiliation strings into unique forms. A frequency analysis for the WoS 251 SCs and check for correspondence with ESI fields was carried out. The subsets were further processed together to derive BC ID cards displaying the most frequent sets of items for each community. The Louvain algorithm that proposes a hierarchy of community partition, with fewer communities and higher modularity, was used to extract communities with 10 or more documents offered by the default option in the software [6].

\section{RESULTS OF THE SECOND STAGE ANALYSIS}

\section{A. Frequency analysis for WoS subject categories (SC)}

Simple frequency analysis of the 3,011 documents for WoS 251 SCs suggests the presence of 200 categories, of which 179 have at least 2 documents. Seventeen SCs, to each of which belong at least $2 \%$ (61) documents, arranged into 10 groups considering their relatedness judging from their correspondence to broad ESI disciplines, are as follows:

- Public, Environmental \& Occupational Health (19.5\%)

- Medicine, General \& Internal (12.7\%); Tropical Medicine (5.6\%); Infectious Diseases (5.4\%); Pediatrics (3.3\%); Surgery (2.7\%); Ophthalmology $(2.5 \%)$

- Environmental Sciences (8.4\%); Ecology (2.5\%)

- Plant Sciences (5\%)

- Geosciences, Multidisciplinary (3.5\%); Geography, Physical $(2.5 \%)$

- $\quad$ Agronomy (3.4\%)

- Water Resources (3.2\%)

- Pharmacology \& Pharmacy $(2.6 \%)$

- Microbiology (2.1\%)

- Biochemistry \& Molecular Biology (2\%)

The most prolific WoS SC is "Public, Environmental \& Occupational Health" with 680 publications (i.e., nearly every fifth) corresponding to as many as 9 ESI fields. Among those 9 fields, assignments to "Clinical Medicine" (with 356 or $59 \%$ documents), "Social Sciences, general" (226 or 37\% documents) and Environment/Ecology (with 16 or $2.6 \%$ documents) are prominent. The share of "Public, Environmental \& Occupational Health" SC corresponding to "Social Sciences, general" ESI field is 7.5\%. This implies that the disciplinary contribution of ca. $8 \%$ of social sciences seen from ESI22 scheme (see Table I) mostly results from this particular SC related to the health sector rather than other traditional social sciences subjects like history. Only a comparative analysis of the disciplinary schemes at various scales, thus, reveals the significance of the percentage contributions of individual subject areas and categories. The importance of research in the health and medical as well as environmental sectors was pointed out also in $[7,8]$.

\section{B. Detection of Bibliographic Communities}

Processing the bibliographic data parsed into individual items by Louvain algorithm limiting to minimum 10 documents yield ID cards for $25 \mathrm{BC}$ communities. A typical ID card for the largest community with 443 documents and the most frequent keyword "management" is given the title of "Environmental (biodiversity, climate, community) management" (Table II). It contains the community characteristics, such as, countries (Nepal, India, China), regions (Himalaya, to which most of Nepal belongs, and Tibet), objects (community forest, land, water, climate, biodiversity), topics of concern (management, deforestation, conservation, land use, impact, traditional knowledge), research fields (environmental science, physical geography, water resources, ecology, geosciences, forestry, plant sciences, meteorology, biodiversity conservation), important journals (e.g., mountain research and development), top contributors (Chinese Acad Sci, Peoples R China; Norwegian Univ Life Sci, Norway; Int Water management Inst, Nepal), authors ( 8 from Nepal, 2 from abroad), and so on. Therefore, the ID card provides an overview of the past research landscape of Nepal covered by this community.

To provide a comprehensive picture on themes covered by these communities, each with 443 (maximum) to 12 (minimum) documents, six "broad groups" based on the author's tentative interpretation are suggested (Tables II, III). Four communities are related with earth, environment and biodiversity (tectonics \& natural hazards; environmental pollution, remediation and conservation; wild-life preservation); 12 communities with medical \& health sciences (maternal \& child health; tropical infectious diseases; cancer \& cardiovascular diseases; mountain sickness; blindness) closely linked also with social sciences; 3 communities with agricultural \& veterinary sciences (pathogens of major crops (maize, wheat, rice) and crops yields, plant genes, and dairy farming); 2 communities with pharmacology and traditional medicine; 3 communities with materials science, and 1 small community of 12 documents with astronomy.

\section{BRIEF DISCUSSION AND CONLUSIONS}

The BC community data provide wider insights into the diversity of research conducted in Nepal. For example, 3 integrative topics (see (i)-(iii) in Table I) correspond to at least 10 communities grouped into "b. Medical and health sciences" (Table III), while the remaining 3 topics (iv)-(vi) in Table I expand to communities in a. \& c. in Table III. In addition, communities under new groups "d. pharmacology and ethnomedicine", "e. materials science", "f. astronomy" have been identified. These new data give details on the diverse items in each community or research cluster. 
TABLE II. AN ID CARD FOR BC COMMUNITY “ENVIRONMENTAL (BIODIVERSITY, CLIMATE, COMMUNITY) MANAGEMENT*

\begin{tabular}{|c|c|c|c|c|c|}
\hline Keyword & $\mathrm{f}(\%)$ & sigma & Institution & $\mathrm{f}(\%)$ & sigma \\
\hline MANAGEMENT & 7.45 & $5 \quad 3.88$ & TRIBHUVAN UNIV, NEPAL & 30.93 & 0.49 \\
\hline NEPAL & 6.55 & 2.89 & INT CTR INTEGRATED MT DEV ICIMOD, NEPAL & 20.77 & 18.22 \\
\hline INDIA & 6.32 & -0.42 & KATHMANDU UNIV, NEPAL & 9.48 & 4.28 \\
\hline CONSERVATION & 5.64 & 6.54 & CHINESE ACAD SCI, PEOPLES R CHINA & 7.67 & 9.03 \\
\hline BIODIVERSITY & 4.51 & 7.97 & NORWEGIAN UNIV LIFE SCI UMB, NORWAY & 3.61 & 5.86 \\
\hline DIVERSITY & 3.84 & 5.3 & INT WATER MANAGEMENT INST, NEPAL & 2.71 & 7.7 \\
\hline HIMALAYA & 3.61 & 5.19 & DEPT HYDROL \& METEOROL, NEPAL & 2.71 & 7.29 \\
\hline CLIMATE-CHANGE & 3.39 & 6.4 & UNIV PADUA, ITALY & 2.26 & 4.74 \\
\hline LAND-USE & 2.71 & 5.78 & UNIV MELBOURNE, AUSTRALIA & 2.26 & 2.77 \\
\hline GROWTH & 2.48 & 1.26 & UNIV BERN, SWITZERLAND & 2.26 & 5.24 \\
\hline VEGETATION & 2.26 & 5.45 & UNIV COPENHAGEN, DENMARK & 2.26 & 5.24 \\
\hline DYNAMICS & 2.26 & 3.47 & ASIAN INST TECHNOL, THAILAND & 2.26 & 4.98 \\
\hline KNOWLEDGE & 2.03 & 4.18 & NEPAL AGR RES COUNCIL, NEPAL & 2.26 & -0.2 \\
\hline IMPACTS & 2.03 & 4.45 & UNIV NAT RES \& LIFE SCI VIENNA, AUSTRIA & 2.03 & 4.83 \\
\hline COMMUNITIES & 2.03 & 4.18 & INT WATER MANAGEMENT INST, SRI LANKA & 2.03 & 6.66 \\
\hline CHINA & 2.03 & 1.16 & POKHARA UNIV, NEPAL & 2.03 & 2.52 \\
\hline CLIMATE & 2.03 & 4.45 & UNIV BERGEN, NORWAY & 1.81 & 2.13 \\
\hline DEFORESTATION & 1.81 & 4.72 & UNIV WAGENINGEN \& RES CTR, NETHERLANDS & 1.81 & 3.89 \\
\hline \multirow[t]{2}{*}{ TIBETAN PLATEAU } & \multirow[t]{2}{*}{1.81} & 1.98 & AGR UNIV, NORWAY & 1.58 & 4.92 \\
\hline & & & FOREST ACT, NEPAL & 1.58 & 5.87 \\
\hline Subject & $\mathrm{f}(\%)$ & sigma & Reference & \multicolumn{2}{|c|}{$f(\%)$ sigma } \\
\hline Environmental Sciences & 27.54 & $+\quad 14.57$ & Shrestha AB, 1999, J CLIMATE (12), 2775 & 4.29 & 9.36 \\
\hline Geography, Physical & 11.74 & 12.62 & Press JR, 2000, ANNOTATED CHECKLIST & 4.06 & 9.43 \\
\hline Water Resources & 11.74 & 10.24 & Manandhar NP, 2002, PLANTS PEOPLE NEPAL & 3.84 & 9.17 \\
\hline Ecology & 11.06 & 11.57 & Shrestha AB, 2000, INT J CLIMATOL (20), 317 & 2.71 & 7.69 \\
\hline Geosciences, Multidisciplinary & 8.35 & 5.65 & Liu XD, 2000, INT J CLIMATOL (20), 1729 & 2.71 & 7.69 \\
\hline Forestry & 7.9 & 12.3 & Ostrom E, 1990, GOVERNING COMMONS EV & 2.71 & 7.29 \\
\hline Plant Sciences & 7.22 & 2.17 & Adhikari B, 2004, ECOL ECON (48), 245 & 2.48 & 7.36 \\
\hline Environmental Studies & 6.32 & 9.35 & Acharya KP, 2002, INT FOREST REV (4), 149 & 2.48 & 7.36 \\
\hline Meteorology \& Atmos Sci & 6.09 & 6.82 & Pilbeam CJ, 2000, AGR ECOSYST ENVIRON (79), 61 & 2.48 & 7.36 \\
\hline \multirow[t]{2}{*}{ Biodiversity Conservation } & \multirow{2}{*}{\multicolumn{2}{|c|}{5.42}} & Brown S, 2000, J ENVIRON MANAGE (59), 217 & 2.26 & 7.02 \\
\hline & & & Nash j E, 1970, J HYDROL (10), 282 & 2.26 & 7.02 \\
\hline Journal & \multirow{2}{*}{\multicolumn{2}{|c|}{$\mathrm{f}(\%) \quad \begin{array}{cc}\text { sigma } \\
7.9 & 11.11\end{array}$}} & Immerzeel WW, 2010, SCIENCE (328), 1382 & 2.03 & 6.66 \\
\hline MT RES DEV & & & Agarwal B, 2001, WORLD DEV (29), 1623 & 2.03 & 6.66 \\
\hline SMALL-SCALE FOR & 2.03 & 6.68 & Mool p K, 2001, INVENTORY GLACIERS G & 2.03 & 6.2 \\
\hline CURR SCI INDIA & 1.58 & 4.94 & Barnett TP, 2005, NATURE (438), 303 & 2.03 & 6.66 \\
\hline ENVIRON MANAGE & \multirow{2}{*}{$\begin{array}{l}1.58 \\
1.58\end{array}$} & 5.89 & Carson B, 1992, 21 ICIMOD & 2.03 & 6.66 \\
\hline J ETHNOBIOL ETHNOMED & & 5.37 & Bhattarai S, 2006, J ETHNOBIOL ETHNOMED (2) & 2.03 & 6.66 \\
\hline FOREST ECOL MANAG & 1.35 & 4.9 & Agrawal A, 2001, WORLD DEV (29), 1649 & 2.03 & 6.66 \\
\hline INDIAN J TRADIT KNOW & 1.35 & 3.74 & Agrawal A, 2001, POLIT SOC (29), 485 & 1.81 & 5.79 \\
\hline CLIMATIC CHANGE & 1.35 & 5.45 & Polunin O, 1984, FLOWERS HIMALAYA & 1.81 & 6.28 \\
\hline INT J SUST DEV WORLD & 1.13 & 4.98 & & & \\
\hline NUTR CYCL AGROECOSYS & 1.13 & 4.98 & & & \\
\hline Country & $\mathrm{f}(\%)$ & sigma & RefJournal & $\mathrm{f}(\%)$ & sigma \\
\hline Nepal & 95.71 & -3.6 & MT RES DEV & 25.73 & 21.54 \\
\hline USA & 14.45 & -2.45 & SCIENCE & 23.93 & 6.91 \\
\hline India & 12.42 & -1.44 & WORLD DEV & 16.48 & 15.75 \\
\hline Peoples R China & 11.29 & 6.89 & NATURE & 16.03 & 3.19 \\
\hline England & 10.38 & 0.68 & P NATL ACAD SCI USA & 12.87 & 2.69 \\
\hline Norway & 7.9 & 5.16 & AGR ECOSYST ENVIRON & 11.74 & 12.32 \\
\hline Japan & 7.67 & -2.18 & ENVIRON MANAGE & 11.29 & 13.48 \\
\hline Germany & 7.45 & 2.65 & CONSERV BIOL & 10.61 & 11.04 \\
\hline Netherlands & 6.09 & 2.92 & BIODIVERS CONSERV & 10.16 & 12.11 \\
\hline Canada & 5.87 & 3.08 & HYDROL PROCESS & 10.16 & 13.4 \\
\hline Title Words & $\mathrm{f}(\%)$ & sigma & Author & $\mathrm{f}(\%)$ & sigma \\
\hline NEPAL & 41.08 & 2.73 & Bajracharya RM & 4.74 & 9.91 \\
\hline MANAGEMENT & 9.71 & 7.97 & Jha PK & 4.51 & 9.65 \\
\hline WATER & 8.13 & 7.25 & Sitaula BK & 4.06 & 9.46 \\
\hline FOREST & 7.67 & 12.09 & Sharma S & 3.61 & 3.48 \\
\hline CASE & 6.32 & 2.79 & Sharma E & 3.39 & 7.93 \\
\hline COMMUNITY & 6.32 & 6.84 & Shrestha BB & 2.26 & 7.04 \\
\hline CHANGE & 6.09 & 8.6 & $\mathrm{Xu} \mathrm{JC}$ & 2.26 & 6.6 \\
\hline ANALYSIS & 5.87 & 3.44 & Innocenti G & 2.03 & 6.68 \\
\hline REGION & 5.64 & 6.11 & Shrestha S & 2.03 & 0.11 \\
\hline & & & Shrestha KK & 2.03 & 6.68 \\
\hline
\end{tabular}

* Number of documents in the community, $\mathrm{N}=443$. Average internal link weight, $\omega_{\text {in }}$ is approximately equal to $1 / 811$. The $\mathrm{BC}$ network was built by keeping a link between articles sharing at least 1 reference. For each item listed under keyword, subject, etc., frequency (f (\%))and the significance parameter sigma $=\operatorname{sqrt}\{\mathrm{N}\}(\mathrm{f}-\mathrm{p}) / \operatorname{sqrt}\{\mathrm{p}(1-\mathrm{p})\}[$ where $\mathrm{N}$ is the number of documents within the community and $\mathrm{f}$ and $\mathrm{p}$ are the proportion of articles respectively within the community and within the database displaying that item] are given. A higher sigma suggests a greater significance, and a value exceeding 5 is really highly significant [6]. 
TABLE III. A BRIEF COMPILATION OF 25 BC COMMUNITIES EXTRACTED BY BIBLIOMETRIC ANALYSIS OF

3,011 DOCUMENTS (ARTICLES, REVIEWS) FROM NEPAL.

\begin{tabular}{|c|c|c|c|c|}
\hline $\begin{array}{l}\text { Broad } \\
\text { group }\end{array}$ & $\begin{array}{l}\text { Comm } \\
\text { unity } \\
\text { ID, } \\
\text { Ndocs }\end{array}$ & $\begin{array}{l}\text { Research title based on } \\
\text { keywords and terms in } \\
\text { document titles }\end{array}$ & $\begin{array}{l}\text { Most frequent followed by } 5 \text { additional } \\
\text { frequent and significant keywords }\end{array}$ & $\begin{array}{l}\text { Lead organizations } \\
\text { (6 largest contributors) }\end{array}$ \\
\hline \multirow{4}{*}{ 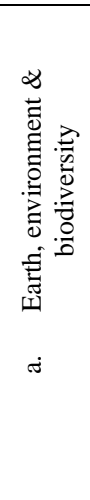 } & $\begin{array}{c}1 \\
(\mathbf{4 4 3})\end{array}$ & $\begin{array}{l}\text { Environmental (biodiversity, } \\
\text { climate, community) } \\
\text { management }\end{array}$ & $\begin{array}{l}\text { MANAGEMENT; CONSERVATION; } \\
\text { BIODIVERSITY; HIMALAYA; } \\
\text { CLIMATE-CHANGE; LAND-USE }\end{array}$ & $\begin{array}{l}\text { Tribhuvan Univ, Nepal; ICIMOD, Nepal; Kathmandu } \\
\text { Univ, Nepal; Chinese Acad Sci, Peoples R China; } \\
\text { Norwegian Univ Life Sci, Norway; Int Water } \\
\text { management Inst, Nepal }\end{array}$ \\
\hline & $\begin{array}{c}4 \\
(\mathbf{1 1 1})\end{array}$ & $\begin{array}{l}\text { Tectonic evolution, } \\
\text { deformation and hazard in } \\
\text { Nepal Himalaya and Tibet }\end{array}$ & $\begin{array}{l}\text { MODEL; GPS MEASUREMENTS; } \\
\text { NEPAL HIMALAYA; TECTONIC } \\
\text { EVOLUTION; MAIN CENTRAL } \\
\text { THRUST; SEISMOTECTONICS }\end{array}$ & $\begin{array}{l}\text { Tribhuvan Univ, Nepal; Dept Mines \& Geol, Nepal; } \\
\text { Kathmandu Univ, Nepal; Commissariat Energie Atom, } \\
\text { France; Kathmandu Univ, Nepal; Univ Paris Diderot, } \\
\text { France }\end{array}$ \\
\hline & $\begin{array}{c}12 \\
(\mathbf{6 3})\end{array}$ & $\begin{array}{l}\text { Atmospheric pollution and } \\
\text { groundwater contamination } \\
\text { in Kathmandu Valley }\end{array}$ & $\begin{array}{l}\text { CONTAMINATION; PARTICULATE } \\
\text { MATTER; EMISSIONS; ADSORPTION; } \\
\text { GROUNDWATER; WASTE-WATER }\end{array}$ & $\begin{array}{l}\text { Tribhuvan Univ, Nepal; Kathmandu Univ, Nepal; } \\
\text { Environm \& Publ Hlth Org, Nepal; Saga Univ, Japan; } \\
\text { Osaka Univ, Japan; Univ Maryland, USA }\end{array}$ \\
\hline & $\begin{array}{c}14 \\
(\mathbf{5 1})\end{array}$ & $\begin{array}{l}\text { Wild-life (Tiger, Rhinoceros, } \\
\text { Panthera, Bird) conservation } \\
\text { in national parks }\end{array}$ & $\begin{array}{l}\text { INDIA; CONSERVATION; NATIONAL- } \\
\text { PARK; POPULATIONS; MORTALITY; } \\
\text { DICLOFENAC }\end{array}$ & $\begin{array}{l}\text { World Wildlife Fund, Nepal; Bird Conservat, Nepal; } \\
\text { Tribhuvan Univ, Nepal; Royal Soc Protect Birds, Nepal; } \\
\text { Wildlife Inst, India; Dept Natl Pk \& Wildlife Conservat, } \\
\text { Nepal }\end{array}$ \\
\hline \multirow{8}{*}{ 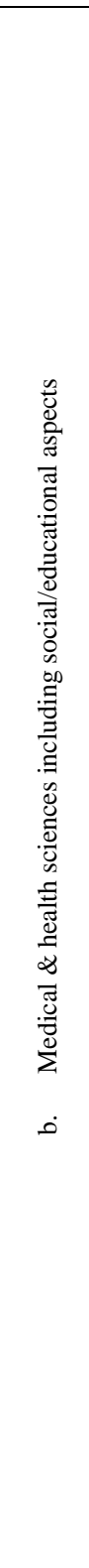 } & $\begin{array}{c}3 \\
(161)\end{array}$ & $\begin{array}{l}\text { Cancer and cardiovascular } \\
\text { diseases: prevalence, risk } \\
\text { factors, prevention and cure }\end{array}$ & $\begin{array}{l}\text { POPULATION; PREVALENCE; } \\
\text { CANCER; MORTALITY; BLOOD- } \\
\text { PRESSURE; CARDIOVASCULAR- } \\
\text { DISEASE }\end{array}$ & $\begin{array}{l}\text { BP Koirala Inst Hlth Sci, Nepal; Tribhuvan Univ, Nepal; } \\
\text { Manipal Coll Med Sci, Nepal; BP Koirala Mem canc } \\
\text { Hosp, Nepal; Nepal Army Inst Hlth Sci, Nepal; } \\
\text { Kathmandu Med Coll, Nepal }\end{array}$ \\
\hline & $\begin{array}{c}7 \\
(\mathbf{1 0 0})\end{array}$ & $\begin{array}{l}\text { Health (mental, sexual, post- } \\
\text { traumatic) disorders in } \\
\text { women \& school children }\end{array}$ & $\begin{array}{l}\text { DISORDERS; HEALTH; HIV/AIDS; } \\
\text { DEPRESSION; POSTTRAUMATIC- } \\
\text { STRESS-DISORDER; MENTAL- } \\
\text { HEALTH }\end{array}$ & $\begin{array}{l}\text { Tribhuvan Univ, Nepal; BP Koirala Inst Hlth Sci, Nepal; } \\
\text { Transcultural Psychological Org, Nepal; Healthnet TPO, } \\
\text { Netherlands; Emory Univ, USA; Univ Tokyo, Japan }\end{array}$ \\
\hline & $\begin{array}{c}8 \\
(95)\end{array}$ & $\begin{array}{l}\text { Typhoid-fever (infectious } \\
\text { disease) in Nepal, India and } \\
\text { Bangladesh }\end{array}$ & $\begin{array}{l}\text { FEVER; CHILDREN; MULTIDRUG- } \\
\text { RESISTANT; PARATYPHI-A; } \\
\text { EPIDEMIOLOGY; SALMONELLA- } \\
\text { TYPHI }\end{array}$ & $\begin{array}{l}\text { Univ Oxford (Hosp Trop Dis, Vietnam), England; Patan } \\
\text { Hosp, Nepal; Tribhuvan Univ, Nepal; CIWEC Clin } \\
\text { Travel Med Ctr, Nepal; Patan Acad Hlth Sci, Nepal; } \\
\text { Armed Forces Res Inst Med Sci, Thailand }\end{array}$ \\
\hline & $\begin{array}{l}15 \\
(\mathbf{5 1})\end{array}$ & $\begin{array}{l}\text { Infectious diseases } \\
\text { (Tuberculosis and Leprosy): } \\
\text { diagnosis and treatment }\end{array}$ & $\begin{array}{l}\text { INDIA; PULMONARY } \\
\text { TUBERCULOSIS; HEALTH; LEPROSY; } \\
\text { EPIDEMIOLOGY; SOCIAL- } \\
\text { PARTICIPATION }\end{array}$ & $\begin{array}{l}\text { Manipal Coll Med Sci, Nepal; Tribhuvan Univ, Nepal; } \\
\text { Natl Tuberculosis Ctr, Nepal; Green Pastures Hosp \& } \\
\text { Rehabil Ctr, Nepal; German Nepal TB Project, Nepal; } \\
\text { World Hlth Org, Switzerland }\end{array}$ \\
\hline & $\begin{array}{c}16 \\
(\mathbf{4 8})\end{array}$ & $\begin{array}{l}\text { High altitude sickness (AMS, } \\
\text { hypoxia and edema): } \\
\text { prevention \& cure }\end{array}$ & $\begin{array}{l}\text { ACUTE MOUNTAIN-SICKNESS; } \\
\text { HYPOXIA; PROPHYLAXIS; } \\
\text { PREVENTION; HIGH-ALTITUDE } \\
\text { ILLNESS; TOURISTS }\end{array}$ & $\begin{array}{l}\text { Nepal Int Clin, Nepal; Himalayan Rescue Assoc, Nepal; } \\
\text { Tribhuvan Univ, Nepal; Univ Sydney, Australia; Univ } \\
\text { Otago, New Zealand; Univ British Columbia, Canada }\end{array}$ \\
\hline & $\begin{array}{l}18 \\
(\mathbf{4 0})\end{array}$ & $\begin{array}{l}\text { Infectious diseases (Dengue, } \\
\text { Japanese encephalitis, } \\
\text { epilepsy): diagnosis, } \\
\text { immunization \& treatment }\end{array}$ & $\begin{array}{l}\text { INFECTION; VIRUS; CHILDREN; } \\
\text { IMMUNIZATION; DENGUE; } \\
\text { JAPANESE ENCEPHALITIS }\end{array}$ & $\begin{array}{l}\text { BP Koirala Inst Hlth Sci, Nepal; Natl Zoonoses \& Food } \\
\text { Hyg Res Ctr, Nepal; Kanti Childrens Hosp, Nepal; Cent } \\
\text { Vet Hosp Tripureshwar, Nepal; Minist Hlth \& Populat, } \\
\text { Nepal; Tribhuvan Univ, Nepal }\end{array}$ \\
\hline & $\begin{array}{l}19 \\
(\mathbf{3 5})\end{array}$ & $\begin{array}{l}\text { Leprosy: diagnosis, } \\
\text { management \& treatment }\end{array}$ & $\begin{array}{l}\text { MYCOBACTERIUM-LEPRAE; } \\
\text { TUBERCULOSIS; SUSCEPTIBILITY; } \\
\text { DIAGNOSIS; NECROSIS-FACTOR- } \\
\text { ALPHA; LEPROMATOUS LEPROSY }\end{array}$ & $\begin{array}{l}\text { Anandaban Hosp, Nepal; London Sch Hyg \& Trop Med, } \\
\text { England; Green Pastures Hosp \& Rehabil Ctr, Nepal; } \\
\text { Univ Washington, USA; Univ Aberdeen, Scotland; Univ } \\
\text { Med \& Dent New Jersey, USA }\end{array}$ \\
\hline & $\begin{array}{l}24 \\
(\mathbf{1 3})\end{array}$ & $\begin{array}{l}\text { Health education, } \\
\text { professionals, policy and } \\
\text { services in Nepal }\end{array}$ & $\begin{array}{l}\text { PHYSICIANS; RETENTION; CARE; } \\
\text { PERCEPTIONS; HEALTH; } \\
\text { PROFESSIONALS }\end{array}$ & $\begin{array}{l}\text { Nick Simons Inst, Nepal; BP Koirala Inst Hlth Sci, } \\
\text { Nepal; Patan Acad Hlth Sci, Nepal; Patan Hosp, Nepal; } \\
\text { Brigham \& Womens Hosp, USA; Kathmandu Med Coll, } \\
\text { Nepal }\end{array}$ \\
\hline
\end{tabular}


TABLE III (continued)

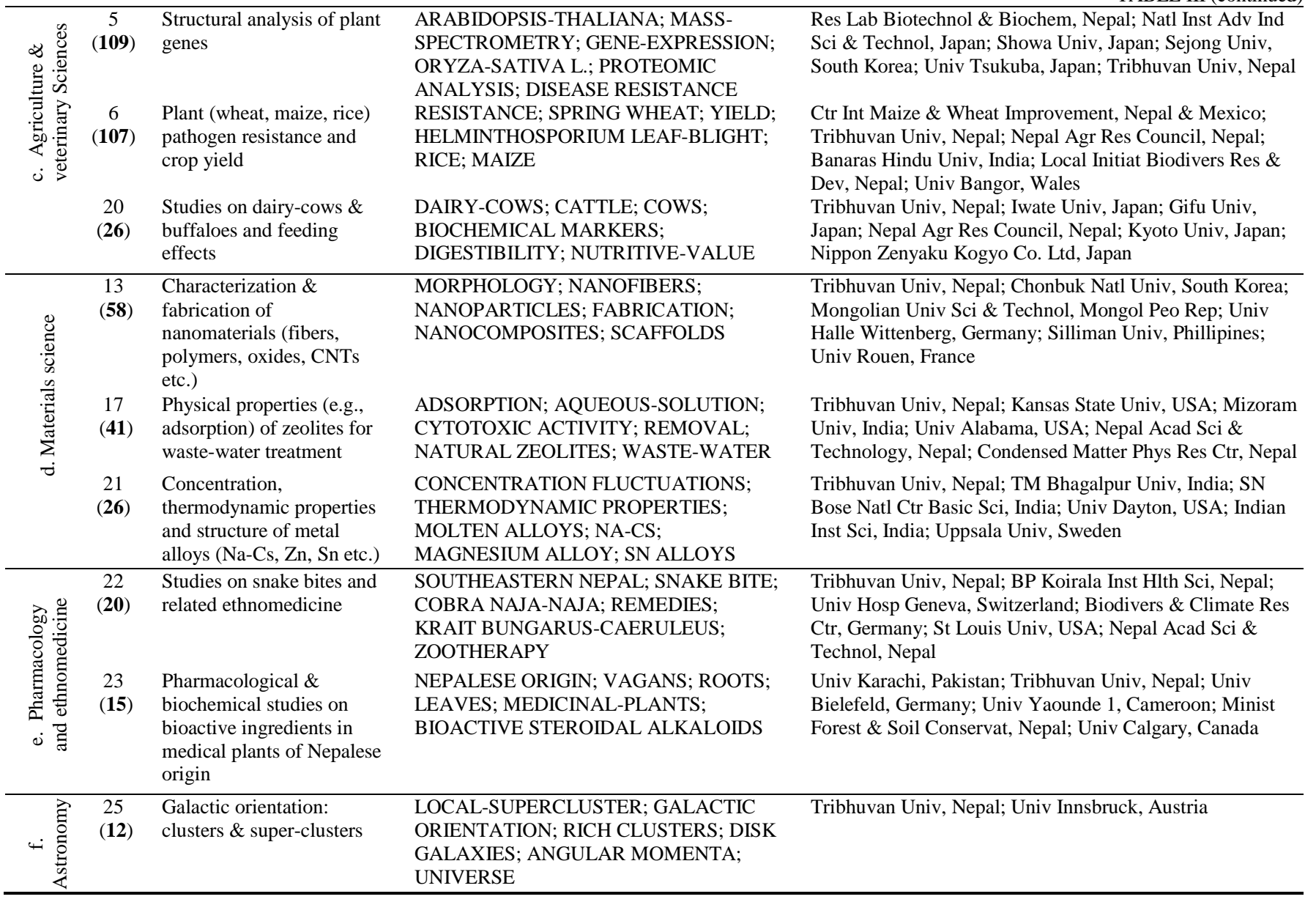

Disciplinary analysis using fine-scale WOS 251 Subject category classification confirms the prominence of research related to environmental \& occupational health.

The BC community data help to identify the academic institutions (e.g., universities and colleges), hospitals, private clinics or projects, governmental research institutes and departments, non-governmental and/or non-profit organizations and charities, both from Nepal and abroad, as significant research contributors. Also, the role of a number of international organizations such as World Wildlife Fund and World Health Organization as research catalysts becomes more evident.

Owing to the threshold of at least 10 documents, and the requirement of at least 1 common reference, this study did not capture smaller communities, which could be important despite the small size. This issue will be examined as this research work progresses further. Also, due to many fields, the titles for communities are tentative, and thus, subject to verification and modification through experts' inputs. Despite this, the BC community data, determined at the level of a country by utilizing fully the internal coupling of documents and their references, complement the information obtained by a broader disciplinary scheme (ESI 22 fields) and impact indicators based on citations and term maps.

\section{REFERENCES}

[1] Web of Science database. http://apps.webofknowledge.com/ (accessed 2017/1/13).

[2] P. Gautam, "An overview of the Web of Science record of scientific publications (2004-2013) from Nepal: focus on disciplinary diversity and international collaboration," unpublished.

[3] B.M. Gupta and A. Bala, "S\&T publications output of Nepal: a quantitative analysis, 2001-10," Scientometrics, 93(3), 2012, pp. 1029-1046.

[4] P. Gautam, "Comparative analysis of scientific publications of research entities using multiple disciplinary classifications," Proc. 2016 IIAI 5th International Congress on Advanced Applied Informatics, Kumamoto, Japan, 2016, pp. 524-528, doi:10.1109/IIAI-AAI.2016.117.

[5] S. Grauwin and P. Jensen, "Mapping scientific institutions," Scientometrics 89, 2011, pp. 943-954.

[6] BiblioTools 2.3 software. Biblio Tool box: Python scripts for scientometric analysis. http://www.sebastian-grauwin.com (accessed 2017/1/13).

[7] P.P. Simkhada, Y.R. Baral. and E.R. van Teijlingen, "Health and medical research in Nepal: a bibliometric review," Asia-Pacific Journal of Public Health 22(4), 2010, pp. 492-500.

[8] A. Gurung and M.L. Bell, "The state of scientific evidence on air pollution and human health in Nepal," Environmental Research, 124, 2013, pp. 54-64. 